\title{
具有聚集诱导发光性能的并吡咯衍生物的多晶性和酸响应行为研究
}

\author{
彭喆季迎春王智佟斌* 石建兵董宇平* \\ (北京理工大学材料学院 结构可控先进功能材料与绿色应用重点实验室 北京 100081)
}

\begin{abstract}
摘要 本工作合成了具有 A-D-A 结构的并吡咯衍生物 4',4"-(2,5-二苯基吡咯并[3,2- $b$ 吡咯-1,4-二基)双([1,1'-联苯]-4腈)(DPPDC), 该化合物具有聚集诱导发光(AIE)特性, 在四氢呋喃/水体系中, 当水含量为 $99 \%$ (体积含量)时, DPPDC 的 荧光强度比在纯四氢呋喃体系中增强了 11 倍. 通过不良溶剂缓慢扩散到溶液体系的方法, 培养得到四种 DPPDC 单晶, 体现出 DPPDC 具有良好的多晶性. 四种晶体发光颜色各不相同, 分别为蓝色、天蓝色、青绿色和绿色, 波长变化范围 为 $29 \mathrm{~nm}$. 此外由于并吡咯结构富含电子特性, 它能与三氟乙酸相互作用, 从而影响其荧光发射, 发光颜色从黄色变为 橙红色，故该化合物可作为酸响应的荧光检测试剂.
\end{abstract}

关键词 多晶性; 并吡咯; 酸响应; 聚集诱导发光

\section{Properties of Polymorphism and Acid Response of Pyrrolopyrrole-based Derivative with Aggregation-induced Emission Behavior}

\author{
Peng, Zhe Ji, Yingchun Wang, Zhi Tong, Bin* Shi, Jianbing Dong, Yuping* \\ (School of Materials Science and Engineering, Beijing Key Laboratory of Construction Tailorable Advanced Functional \\ Materials and Green Applications, Beijing Institute of Technology, Beijing 100081)
}

\begin{abstract}
A new A-D-A type pyrrolopyrrole-based derivative 4',4"-(2,5-diphenyl-pyrrolo[3,2-b]pyrrole-1,4-diyl)bis ([1,1'-biphenyl]-4-carbonitrile) (DPPDC) was synthesized via Suzuki coupling reaction between 1,4-bis(4-bromophenyl)-2,5-diphenyl-1,4-dihydropyrrolo[3,2-b]pyrrole and 4-cyanophenylboronic acid. The fluorescent emission intensities of DPPDC in pure THF solution and lower fraction of water $\left(\varphi_{\mathrm{H} 2 \mathrm{O}} \leqslant 60 \%\right)$ mixtures were weak at around $550 \mathrm{~nm}$. When $\varphi_{\mathrm{H} 2 \mathrm{O}}$ was $99 \%$ in $\mathrm{THF} / \mathrm{H}_{2} \mathrm{O}$ mixtures, the emission was enhanced and blue-shifted at around $505 \mathrm{~nm}$. The maximal fluorescent emission intensity of DPPDC was 11 times higher than that of in pure THF solution, indicating DPPDC exhibiting AIE property. It was also found that four different kinds of crystal structures of DPPDC was cultivated from $\mathrm{CHCl}_{2}-\mathrm{Hexane}, \mathrm{CHCl}_{3}-\mathrm{Hexane}$ and $\mathrm{CHCl}_{3} / \mathrm{Acetone}-\mathrm{Hexane}$ systems via solvent slow diffusion method. Four crystals respectively emitted blue, azure, green and turquoise at 467, 483, 496 and $493 \mathrm{~nm}$, which manifested the polymorphism-dependent fluorescent emission property of DPPDC. Additionally, trifluoroacetic acid (TFA) could make the emitting color change from yellow to orange-red with as-prepared paper containing DPPDC due to the acid-base interaction. The obvious emitting color change of DPPDC can be used as a visual sensor to detect acid gas.

Keywords polymorphism; pyrrolopyrrole; acid response; aggregation-induced emission
\end{abstract}

\section{1 引言}

近年来，有机苂光晶体材料由于在有机发光二极 管、有机场效应晶体管、液晶显示、光波导、刺激响应 等方面具有重要的应用前景已引起人们的广泛关注和 研究兴趣 ${ }^{[1]}$. 有机苂光晶体材料通常以固态的形式被研 究和应用, 多晶性作为有机苂光晶体材料的研究热点之 一, 其原因在于分子的不同构象与堆叠方式使得分子间 具有不同的相互作用, 进而造成不同的宏观性能. 张红 雨等 ${ }^{[2]}$ 报道了一种含并噻唑的 $\pi$ 共轭化合物, 具有四种 晶体结构, 其苂光发射分别为绿色 $\left(\lambda_{\mathrm{em}}=522 \mathrm{~nm}\right)$ 、黄绿 $\left(\lambda_{\mathrm{em}}=561 \mathrm{~nm}\right)$ 、黄色 $\left(\lambda_{\mathrm{em}}=566 \mathrm{~nm}\right)$ 和橘色 $\left(\lambda_{\mathrm{em}}=583 \mathrm{~nm}\right)$, 其中晶体 $\mathrm{A}$ 具有放大自发射(Amplified Spontaneous Emission, ASE) 特性. 田文晶等 ${ }^{[3]}$ 报道了一种具有力致 变色效应的蒽衍生物 BP2VA 化合物, 得到了三种晶体 结构, 其发射的苂光从绿色 $\left(\lambda_{\mathrm{em}}=527 \mathrm{~nm}\right)$ 到橙色 $\left(\lambda_{\mathrm{em}}=\right.$ $579 \mathrm{~nm})$ 再到红色 $\left(\lambda_{\mathrm{em}}=618 \mathrm{~nm}\right)$, 通过研磨或者外部施 加压力可表现出力致变色苂光性能. Park 等 ${ }^{[4]}$ 报道了一 种具有两种晶体结构的化合物 DBDCS，其发射的绿光 $\left(\lambda_{\mathrm{em}}=533 \mathrm{~nm}\right)$ 和蓝光 $\left(\lambda_{\mathrm{em}}=458 \mathrm{~nm}\right)$ 两种苂光可以根据 压力、温度和溶剂气氛等条件进行相互转化. 上述多晶 性的分子含有较大的 $\pi$ 共轭基团, 在不同的晶体结构中,

\footnotetext{
*E-mail: tongbin@bit.edu.cn; chdongyp@bit.edu.cn; Tel.: 010-68917390; Fax: 010-68917390 Received August 13, 2016; published November 29, 2016.

Supporting information for this article is available free of charge via the Internet at http://sioc-journal.cn.

Project supported by the National Basic Research Program of China (973 Program: 2013CB834704) and National Natural Science Foundation of China (Nos: 51328302, 21474009).

项目受科技部国家基础研究计划 973 项目(2013CB834704)和国家自然科学基金(Nos.: 51328302, 21474009)资助.
} 
$\pi$ 共轭程度越大，晶体的发光越红移.

传统的苂光材料由于聚集诱导淬灭效应使得其固 态应用领域受到较大限制. 2001 年, 唐本忠院士 ${ }^{[5]}$ 在研 究六苯基噻咯的发光行为时提出了聚集诱导发光 (Aggregation-Induced Emission, AIE) 概念, 为有机苂光 晶体材料在固态条件下的应用提供了良好的契机. 目 前, AIE 分子在荧光生物成像、化学传感检测、光电器 件等领域的研究已引起人们广泛的关注 ${ }^{[6]}$. 同时, 研究 表明, AIE 分子的作用机理是分子内运动受限, 这类分 子通常具有扭曲的分子构象, 在溶液中由于分子单键旋 转或者分子振动导致能量耗散使其发光较弱, 在聚集态 (如固态, 晶态)时分子单键旋转或者分子振动受到限制, 激发态的能量以辐射的方式耗散, 从而利于苂光在固态 下的发射 ${ }^{[7]}$.

目前, 研究者们对具有多晶性的 AIE 物质进行了大 量的报道. 唐本忠等 ${ }^{[8]}$ 报道了一类具有 AIE 性质的蝴蝶 状二氢葸衍生物, 其中 TDHA 由于在两种晶态与无定型 之间的转换, 使其具有力致、溶致和热致变色特性. 马 於光等 ${ }^{[9]}$ 报道了具有 AIE 特性的氰基取代的二苯乙烯衍 生物 CN-DSB, 它具有两种不同荧光发射的晶体结构, 通过热 $\left(25 \sim 225{ }^{\circ} \mathrm{C}\right)$ 和压力 $(0.04 \sim 2.04 \mathrm{GPa})$ 的处理可以 使 CN-DSB 发射蓝色和绿色荧光. 王悦等 ${ }^{[10]}$ 报道了具有 AIE 特性的席夫碱化合物 SADA, 它能形成两种片状晶 体, 分别发射黄色和红色苂光, 它们都具有 ASE 特性. 上述报道的 AIE 分子的多晶性通常是两种或者三种晶 体结构以及晶态与无定型态之间的研究, 然而具有四种 及四种以上不同晶体结构的 AIE 物质罕见报道.

本工作合成了以并吡咯为电子给体, 氰基作为电子 受体的 A-D-A 类型化合物 4',4"-(2,5-二苯基吡咯并 [3,2- $b]$ 吡咯-1,4-二基)双([1,1'-联苯]-4-腈)(DPPDC), 通 过不良溶剂缓慢挥发进入溶液体系的方法得到四种单 晶结构, 其中两种晶体结构中分别含有两种不同的分子 构象, 通过单晶结构的分析探究了分子聚集态结构对 AIE 性能的影响. 同时, 由于并吡咯核富含电子, 易与 三氟乙酸相互作用, 因此该物质有望作为荧光探针实现 对酸气体的响应与检测.

\section{2 结果与讨论}

\subsection{DPPDC 的合成}

目标产物 DPPDC 的合成路线如图式 1 所示. 中间 产物 1 的合成如文献报道方法 ${ }^{[11]}$. DPPDC 由 1 与 4-氰基 苯硼酸经 Suzuki 偶联反应制得, 经过核磁共振谱和质谱 对目标产物 DPPDC 进行结构确证.

\subsection{DPPDC 光学性质表征}

DPPDC 在 THF 溶液 $\left(10^{-5} \mathrm{~mol} / \mathrm{L}\right)$ 中的紫外可见吸收 谱图在支持文献中如图 S1 所示, DPPDC 的最大吸收峰 在 $333 \mathrm{~nm}$, 它归属于 $\pi-\pi *$ 跃迁吸收带. 为了验证
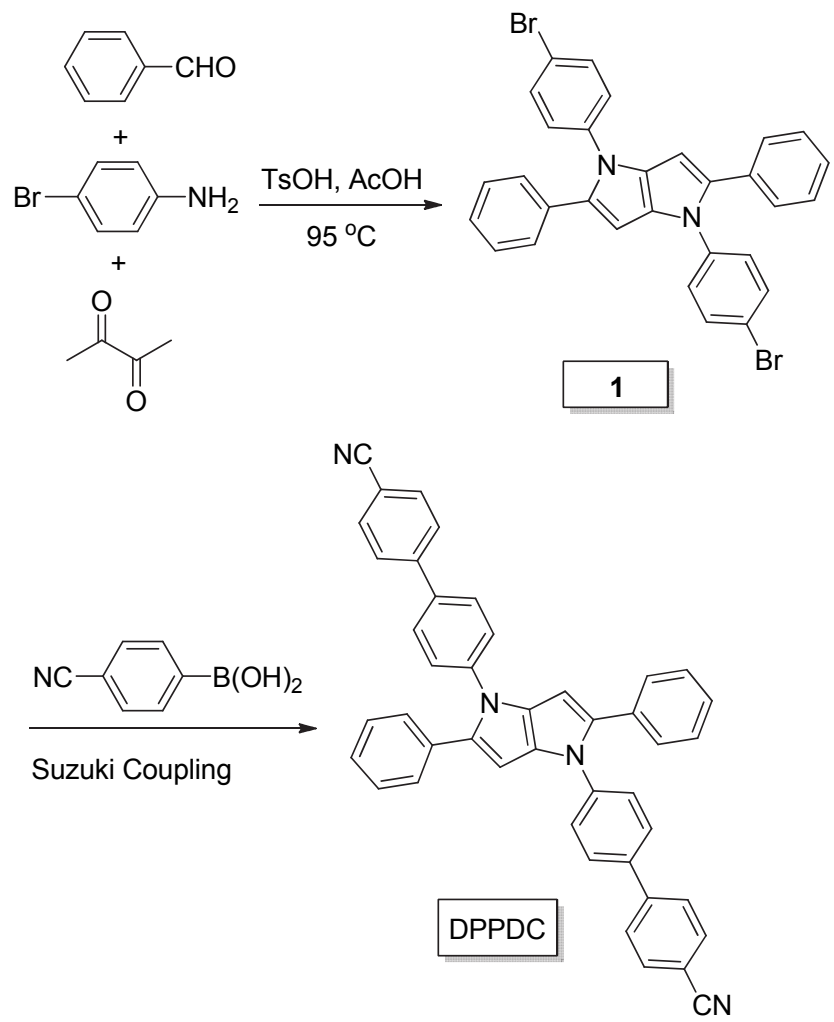

图式 1 DPPDC 的合成路线

Scheme 1 Synthetic routes of DPPDC

DPPDC 的 AIE 性质, 我们对 DPPDC 在 THF $/ \mathrm{H}_{2} \mathrm{O}$ 混合 体系进行了菼光性质的测试. 如图 1 所示, 在纯 THF 中, DPPDC 的发射较弱, 发射波长为 $557 \mathrm{~nm}$, 随着水的加 入, 其苂光强度降低, 当水含量 $\left(\varphi_{\mathrm{H} 2 \mathrm{O}}\right)$ 小于 $60 \%, \mathrm{DPPDC}$ 的荧光淬灭，当 $\varphi_{\mathrm{H} 2 \mathrm{O}}$ 大于 $60 \%$ 时，其荧光强度开始增强， 当 $\varphi_{\mathrm{H} 2 \mathrm{O}}$ 为 $99 \%$ 时，其荧光发射强度达到最大值，是其四 氢呋喃溶液的 11 倍. $\mathrm{H}_{2} \mathrm{O}$ 是 DPPDC 的不良溶剂, 在高 的含水混合体系, $\mathrm{H}_{2} \mathrm{O}$ 会使 DPPDC 形成纳米聚集体，如 图 S2 所示, 在 $90 \% \mathrm{H}_{2} \mathrm{O} / 10 \% \mathrm{THF}$ 体系中, 由动态光散 射实验测得 DPPDC 的聚集体尺寸为 $198.4 \mathrm{~nm}$, 这表明 了聚集体的形成，说明在高的水含量体系中 DPPDC 的 苂光增强是由于分子形成聚集体造成的. 由于 DPPDC 的分子属于 A-D-A 类型，当水含量增加时，混合体系的 极性增加，发射波长稍微红移，苂光强度减弱，属于扭 曲分子内电荷转移(TICT)现象，当水含量超过 $60 \%$ 时， DPPDC 开始形成聚集体，荧光发射不断增强，属于 AIE 效应.

\subsection{DPPDC 的四种晶体结构}

由于 DPPDC 具有 AIE 特性, 我们对 DPPDC 的晶 体结构进行了苂光性质的研究. 根据溶剂缓慢扩散方 法，我们在不同良溶剂一不良溶剂体系中共培养得到四 种不同的晶体结构，它们分别为：二氯甲烷一正己烷体 系中得到晶体命名为 DPPDC1, 三氯甲烷-正己烷体系 中得到晶体命名为 DPPDC2 和 DPPDC3, 三氯甲烷/丙 

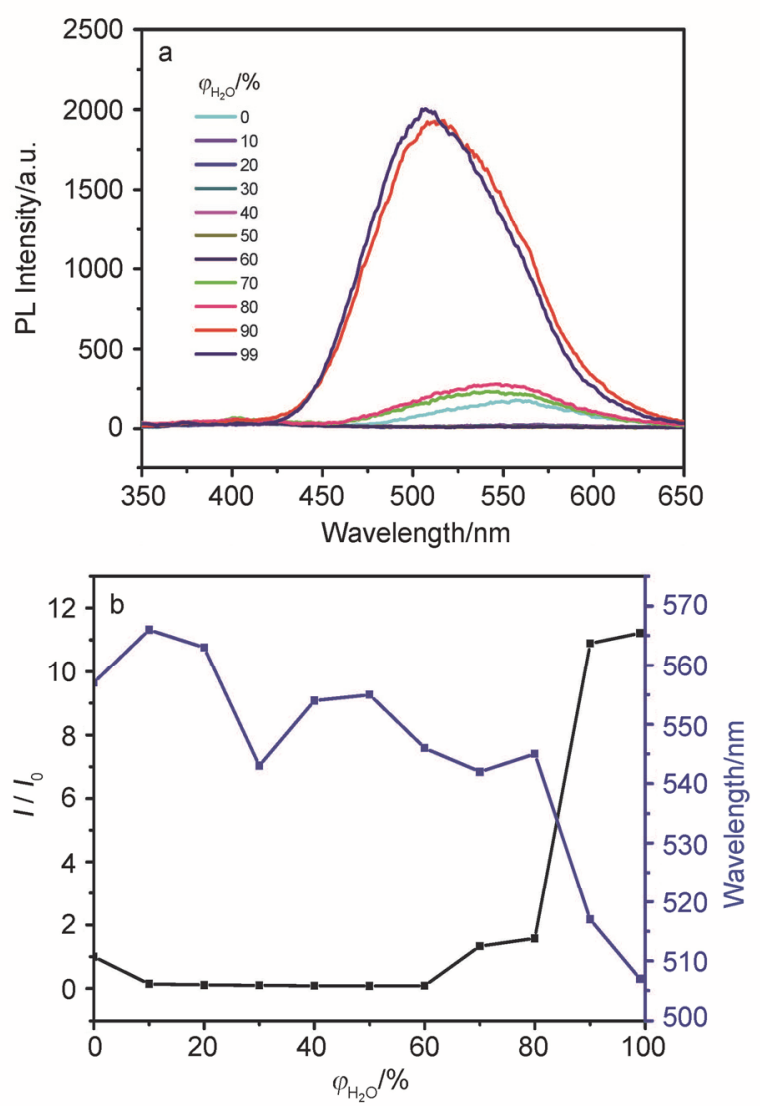

图 1 (a) DPPDC 在四氢呋喃/水体系中不同水含量 $\left(\varphi_{\mathrm{H} 2 \mathrm{O}}\right)$ 下的苂光发 射光谱图; (b) 最大发射强度 $(I)$ 与纯 THF 溶液体系初始最大发射强度 $\left(I_{0}\right)$ 的比值和发射波长与不同水含量的关系图. (浓度: $10^{-5} \mathrm{~mol} / \mathrm{L}$, 激 发波长: $330 \mathrm{~nm}$ )

Figure 1 (a) The emission spectrum of DPPDC in THF/ $\mathrm{H}_{2} \mathrm{O}$ with different water fraction $\left(\varphi_{\mathrm{H} 2 \mathrm{O}}\right)$; (b) Plots of ratio between maximum fluorescence intensity and initial fluorescence intensity and emission wavelength vs water fraction. (Concentration: $10^{-5} \mathrm{~mol} / \mathrm{L}, \lambda_{\mathrm{ex}}=330 \mathrm{~nm}$ )

酮-正己烷体系中得到晶体命名为 DPPDC4. DPPDC1 在 $467 \mathrm{~nm}$ 处发射蓝色苂光, DPPDC2 在 $483 \mathrm{~nm}$ 处发射天蓝 色荧光, DPPDC3 和 DPPDC4 在 496 和 $493 \mathrm{~nm}$ 处发绿色 和青绿色苂光, 它们的苂光发射谱如图 2a 所示, 它们的 苂光发射照片如图 $2 \mathrm{~b}$ 所示. 四种晶体的晶胞参数如表 S1 所示.

图 3 为 DPPDC1 的晶体结构, 在其晶体中分子存在 两种构象形式, 分别命名为 DPPDC1-p 和 DPPDC1-b. 两种构象分子的中心并吡咯核与周围的苯环有着较大 的二面角, DPPDC1-p 的中心并吡咯平面与 1 位 2 位苯环 所在平面的夹角为 $38.01^{\circ}$ 和 $42.27^{\circ}$, 吡咯 1 位上相连的 两个苯环平面的夹角为 $32.40^{\circ}$, DPPDC1-b 相应的三个 角分别为 $39.62^{\circ}, 41.1^{\circ}$ 和 $39.14^{\circ}$. 由于 DPPDC1-b 的并吡 咯 1 位上相连两个苯环之间的夹角大于 DPPDC1-p 的两 个夹角, 所以使得分子 DPPDC1-b 的扭曲程度大于 DPPDC1-p 的扭曲程度. 如图 3b 所示, DPPDC1-p 和 DPPDC1-b 之间存在着大量的氢键-CN $\cdots \cdot \mathrm{HC}$-, 氧基氮
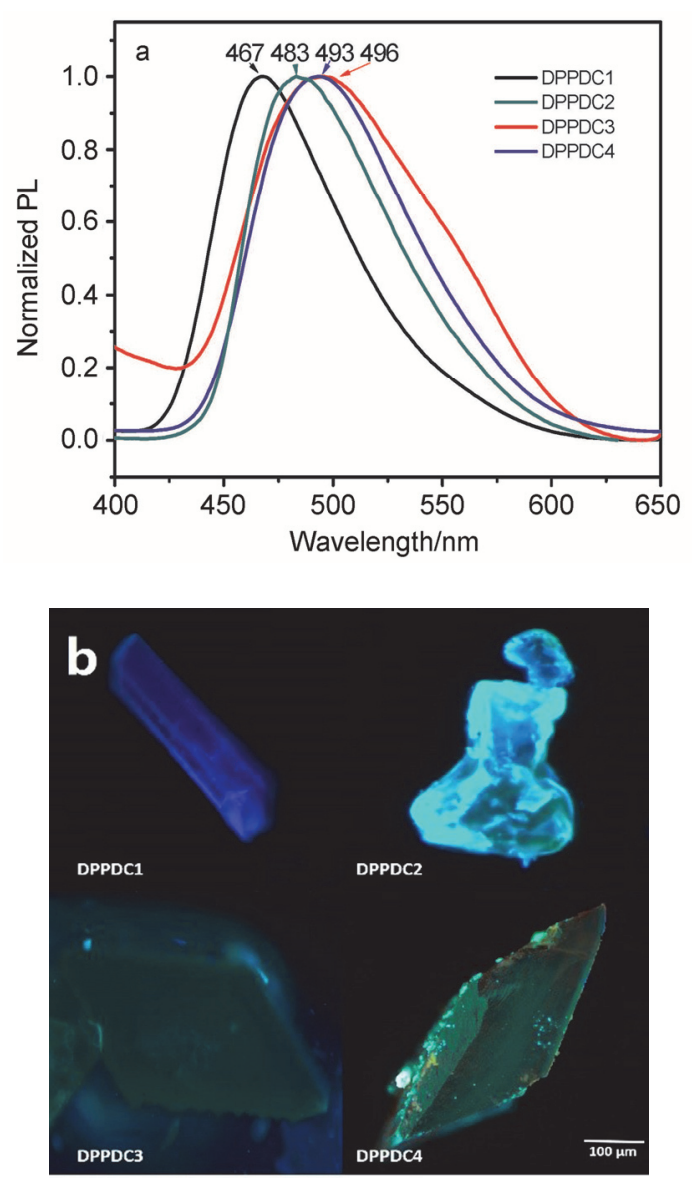

图 2 (a) 四种 DPPDC 晶体的荧光发射谱; (b) 四种 DPPDC 晶体的苂 光照片. (激发波长: $330 \mathrm{~nm}$ )

Figure 2 (a) The emission spectrum of DPPDC1, DPPDC2, DPPDC3 and DPPDC4; (b) The fluorescent image of four DPPDC solids. $\left(\lambda_{\mathrm{ex}}=330\right.$ $\mathrm{nm})$

与苯环氢的距离分别为 $2.537 、 2.610 、 2.680 、 2.737$ 和 $3.015 \AA$. 同时存在着大量的 $\mathrm{CH} \cdots \pi$ 键, 其距离分别为 $2.303 、 2.775 、 2.900 、 2.906 、 3.394$ 和 $3.572 \AA ;$ DPPDC1-p 的并吡咯 1 位上的苯环之间的平行距离为 $3.253 \AA$, 表 明 DPPDC1-p 之间存在一定的 $\pi \cdots \pi$ 相互作用(图 3c), 但 是 DPPDC1-b 之间不存在明显的 $\pi \cdots \pi$ 相互作用. 分子 间存在大量的氢键, $\mathrm{CH} \cdots \pi$ 键, 使得 DPPDC 在聚集态 (固体, 晶体)时, 分子的运动受到限制, 激发态的能量 以苂光的形式释放出来, 最终使得 DPPDC 表现出了 AIE 特性.

由于 DPPDC1 中有两种分子构象，我们设想能否用 不同的溶剂体系培养出只有一种构象分子的晶体. 我们 选择二氯甲烷/甲苯一正己烷，二氯甲烷/甲醇-正己烷， 二氯甲烷/丙酮一正己烷，三氯甲烷一正己烷，三氯甲烷甲苯/正己烷, 三氯甲烷/甲醇-正己烷, 三氯甲烷/丙酮一 正己烷体系. 最终成功拿到晶体的溶剂体系是三氯甲 烷-正己烷和三氯甲烷/丙酮-正己烷. 

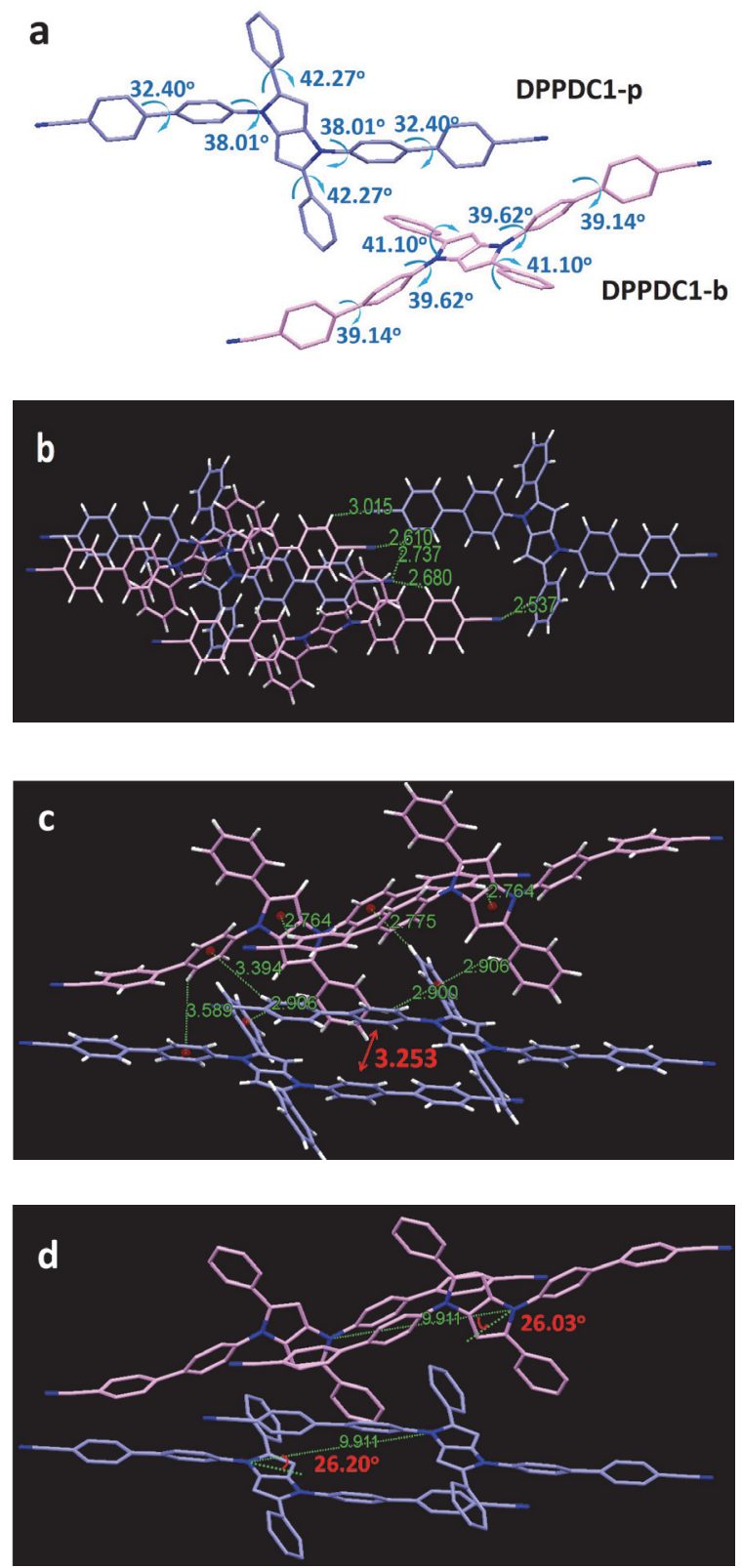

图 3 (a) 晶体 DPDPC1 的晶体结构示意图; (b) DPPDC1 分子间氢键; (c) DPPDC1 分子间的 $\mathrm{CH} \cdots \pi$ 和 $\pi \cdots \pi$ 相互作用; (d) 并吡咯环之间的 距离和倾斜角度

Figure 3 (a) the crystal structure of DPPDC1; (b) H-bonding and (c) $\mathrm{CH}^{\cdots} \pi$ and $\pi \cdots \pi$ interaction of DPPDC1; (d) the distances and tilted angles between pyrrolopyrrole ring of DPPDC1

在三氯甲烷-正己烷体系中得到晶体的苂光显微镜 照片如图 S3 所示, 其中存在发天蓝色和发绿色苂光的 两种晶体, 通过单晶解析验证它们是两种不同的晶体结 构, 分别命名为 DPPDC2 和 DPPDC3. 图 4 为 DPPDC2 的晶体结构. DPPDC2 中分子空间排列和堆叠与 DPPDC1 中的两种分子构象都不同. DPPDC2 的并吡咯 环 $2,2^{\prime}$ 位置的苯环与并吡咯环之间夹角为 $32.21^{\circ} \sim$ $38.75^{\circ}$, 空间位置存在一定的扰动. DPPDC 的并吡咯环
平面与 $2,2^{\prime}$ 位置上两个的苯环之间的夹角分别为 $44.12^{\circ}$ 和 $31.05^{\circ}$. 说明分子内也存在较大的扭曲. 图 4(b)中表 明分子间存在着大量的氢键和 $\mathrm{CH} \cdots \pi$ 键，氰基与吡咯 环 $3,3^{\prime}$ 位置氢的距离为 $2.498 \AA, \mathrm{CH} \cdots \pi$ 距离为 2.679 和 $2.962 \AA$. 图 4(c) DPPDC2 的并吡咯环之间的角度为 $34.58^{\circ}$, 表现为 $\mathrm{J}$-聚集，分子间无 $\pi \cdots \pi$ 相互作用，所以 DPPDC2 发射明亮的天蓝色苂光.
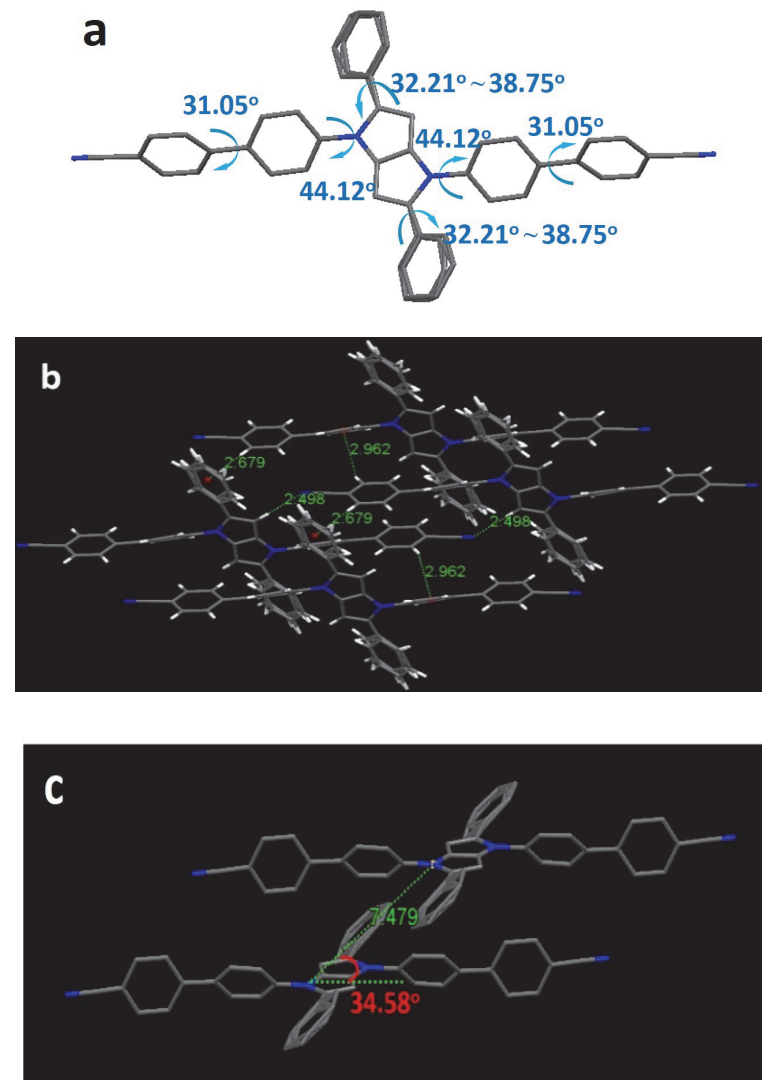

图 4 (a) DPPDC2 晶体结构示意图; (b) DPPDC2 分子间的氢键和 $\mathrm{CH} \cdots \pi$ 相互作用; (c) DPPDC2 分子中并吡咯环间的距离与角度

Figure 4 (a) the crystal structure of DPPDC2; (b) H-bonding and $\mathrm{CH} \cdots \pi$ interaction of DPPDC2; (c) the distance and tilted angle between pyrrolopyrrole ring of DPPDC2

DPPDC3 的晶体结构如图 5 所示, 晶胞中分子只有 一种构象, 但与前面两种晶体的分子构象明显不同. 并 吡咯环平面与 1 位 2 位苯环所在平面的四个夹角都不一 样, 分别为 $47.47^{\circ}, 40.13^{\circ}, 40.33^{\circ}$ 和 $33.71^{\circ}$, 吡咯环 1 位 上两个相连苯环平面的夹角为 $43.64^{\circ}$ 和 $33.79^{\circ}$, 这表明 DPPDC3 晶体中分子空间构象很扭曲. DPPDC 分子间存 在着四种氢键 $($ 氢键距离为 $2.465,2.766,2.785$ 和 3.026 $\AA)$, DPPDC 分子间存在着大量的 $\mathrm{CH} \cdots \pi$ 相互作用, $\mathrm{CH} \cdots \pi$ 距离为 $2.730,3.131,3.043,3.098,3.185$ 和 3.235 Å(图 5b).

DPPDC4 的晶体结构如图 S4 所示, 晶体中存在两 种分子构象, 分子内的夹角 $\left[41.25^{\circ} 、 39.49^{\circ} 、 42.88^{\circ}\right.$ 、 
$41.40^{\circ} 、 38.36^{\circ}$ 和 $32.62^{\circ}$ (图 S4a)]、分子间的氢键 $[2.546$ 、 2.616、2.680、3.022 和 $3.207 \AA$ (图 S4b)]、CH $\cdots \pi$ 相互 作用 $(2.782 、 2.798 、 2.920 、 2.927 、 3.419$ 和 $3.633 \AA)$ 和 $\pi \cdots \pi$ 相互作用 $[3.268 \AA$ (图 S4c)] 与 DPPDC1 很接近, 但 是 DPPDC4 的荧光发射比 DPPDC1 红移了 $26 \mathrm{~nm}$.
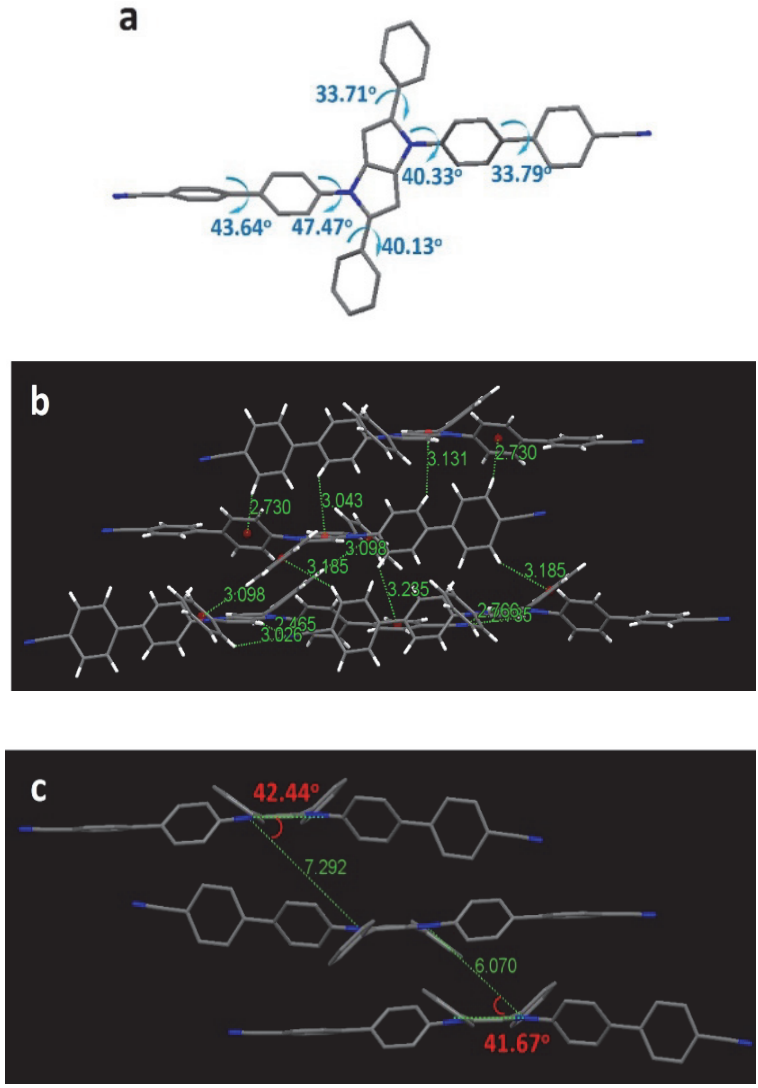

图 5 (a) DPPDC3 的晶体结构示意图; (b) DPPDC3 分子间的氢键和 $\mathrm{CH} \cdots \pi$ 相互作用; (c) DPPDC3 分子中并吡咯环间的距离与角度 Figure 5 (a) the crystal structure of DPPDC3; (b) H-bonding and $\mathrm{CH} \cdots \pi$ interaction of DPPDC3; (c) the distances and tilted angles between pyrrolopyrrole ring of DPPDC3

从上面的晶体结构分析可知, 三氯甲烷-正己烷体 系培养得到的晶体结构(DPPDC2 和 DPPDC3)比较特殊, 一种溶液体系中能培养得到两种不同的晶体结构, 类似 的行为也有文献报道 ${ }^{[12]}$. DPPDC2 并吡咯环 2,2'-位置苯 环空间位置不稳定, 在一定范围内摆动, DPPDC3 分子 空间构象不对称, 各苯环之间扭曲程度不一样. 这可能 与体系中存在的溶剂极性梯度、温度梯度等因素有关. DPPDC1, DPPDC2, DPPDC3 分子的并吡咯环之间的距 离逐渐减小, 分别为 9.911 、7.479 和 7.292 与 $6.070 \AA$, 并 吡咯环之间的倾斜角度分别为 $26.03^{\circ}\left(26.20^{\circ}\right), 34.58^{\circ}$ 和 $42.44^{\circ}\left(41.67^{\circ}\right)$, 倾斜角度逐渐增大(图 3d, 图 4c, 图 5c), 导致分子排列趋于规整, 发光波长红移.

\subsection{DPPDC 的酸响应行为}

由于并吡咯被认为是 $10 \pi$ 电子体系中电子给体能力 最强的的功能单元 ${ }^{[13]}$, 所以在酸性环境中, 酸碱相互作
用对并吡咯化合物的发光行为会造成影响. 我们在前期 工作中研究发现四苯基并吡咯(DPPTP)对三氯甲烷有苂 光淬灭的响应，原因是三氯甲烷在光照条件下分解成氯 化氢气体与 DPPTP 相互作用使其苂光淬灭 ${ }^{[14]}$. DPPDC 在并吡咯环 1,1 '位置上引入了吸电子基团, 我们测试了 DPPDC 对酸的响应性能. 将 DPPDC $\left(10^{-2} \mathrm{~mol} / \mathrm{L}, 50 \mu \mathrm{L}\right)$ 滴到滤纸条, 烘干后置于三氟乙酸(TFA) 的氛围中, DPPDC 的发光立即由原来的黄色变为橙红色, 发光由 $507 \mathrm{~nm}$ 红移至 $572 \mathrm{~nm}$, 当 DPPDC 重新用三乙胺熏蒸 时，DPPDC 的荧光发射峰又变回到 $507 \mathrm{~nm}$, 这表明 DPPDC 对 TFA 具有可逆性响应(图 6). 因此 DPPDC 可 作为三氟乙酸气体的可视化检测. a
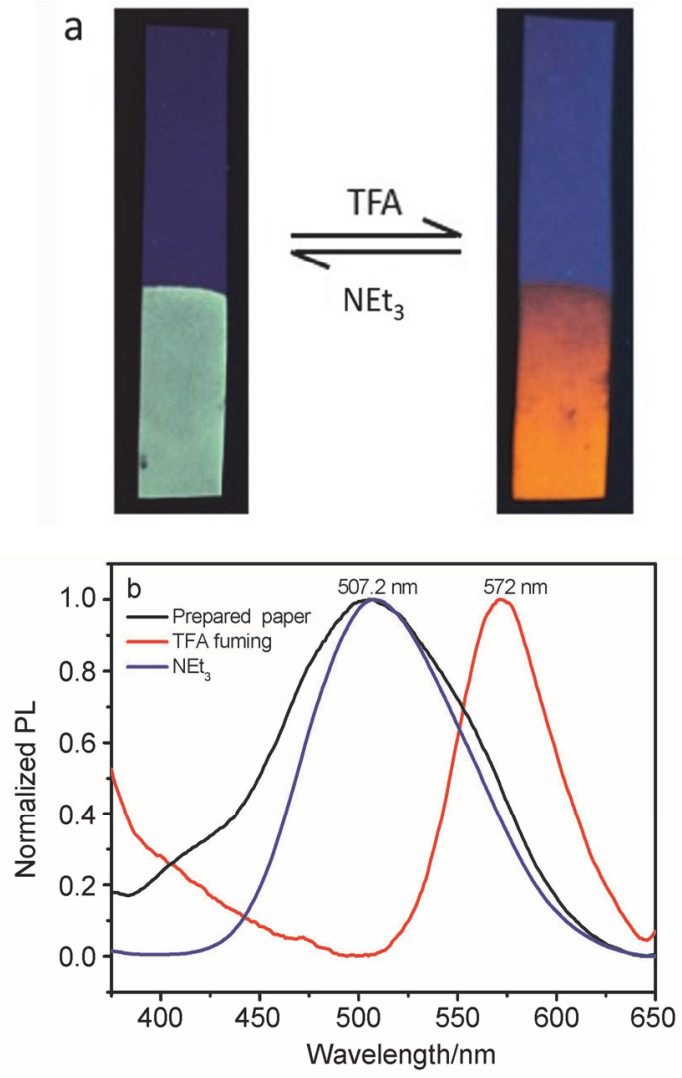

图 6 (a) 含 DPPDC 滤纸条在三氟乙酸和三乙胺熏蒸下苂光颜色变化 图; (b) 含 DPPDC 滤纸条在三氟乙酸和三乙胺熏蒸后的苂光归一化发 射谱图

Figure 6 (a) The change of emission of DPPDC paper fumed by TFA and $\mathrm{NEt}_{3}$; (b) The normalized PL emission spectra of DPPDC paper fumed by TFA and $\mathrm{NEt}_{3}$

为了验证三氟乙酸对 DPPDC 发光行为造成影响的 原因, 我们进行了核磁氢谱的测试, 如图 7 所示, 图 7a 为 DPPDC 在 $\mathrm{CDCl}_{3}$ 中的 ${ }^{1} \mathrm{H}$ NMR 谱. 当加入 $30 \mu \mathrm{L}$ 的 TFA, 可以看出, $\delta 6.5$ 处的峰向低场移至 6.65 和 6.80 , 峰 高变低, 同时 $\delta$ 为 7.3 到 7.7 之间的峰变低变宽, 说明 TFA 对并吡咯环上的氢原子以及直接相连的四个苯环 上的氢的化学位移及峰高影响较大. 并吡咯 1,1 '位置上 相连的两个苯环之间由于存在较大的夹角, 因此对与腈 
基相连的苯环的氢的化学位移影响较小, 所以 $\delta 7.75$ 处 的峰较高. 故此我们推测三氟乙酸与并吡咯环的氮相互 作用, 形成新的化合物, 由于吸电子作用, 使其氢的化

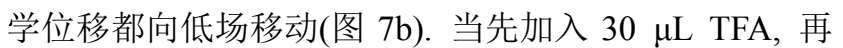
加入 $40 \mu \mathrm{L}$ 的 $\mathrm{NEt}_{3}, \mathrm{DPPDC}$ 的氢的化学位移基本回到 原来的位置, 只有略向低场移动, 这可能是由于为中和 三氟乙酸, 过量的三乙胺导致整个环境呈现弱碱性, 导 致化学位移向低场移动, 这与文献报道结果相一致 ${ }^{[15]}$. 以上研究结果表明 DPPDC 对 TFA 的响应具有可逆性.

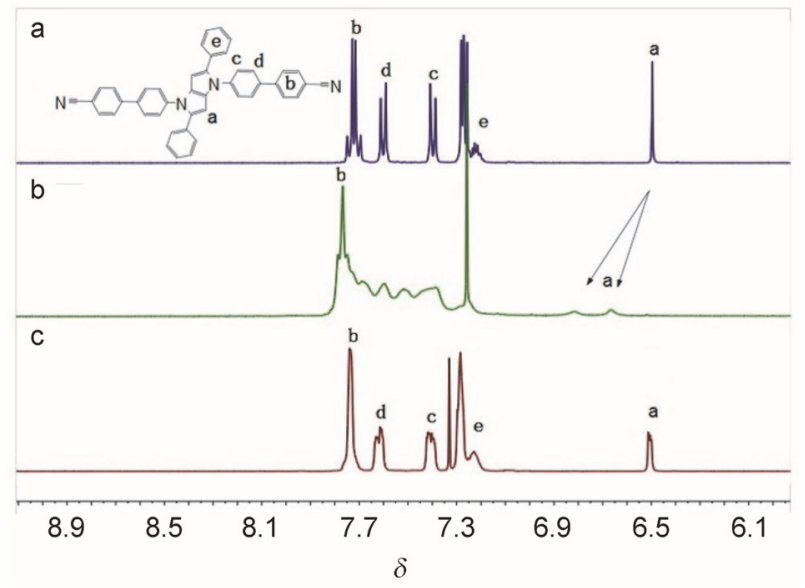

图 $7 \mathrm{DPPDC}$ 在 $\mathrm{CDCl}_{3}$ 加入 $0(\mathrm{a}), 30 \mu \mathrm{L}$ 三氟乙酸(b)以及加入 $30 \mu \mathrm{L}$ 三氟乙酸 $+40 \mu \mathrm{L}$ 三乙胺(c)的核磁氢谱谱图

Figure 7 The ${ }^{1} \mathrm{H}$ NMR spectra of DPPDC in $\mathrm{CDCl}_{3}$ containing (a) 0 , and (b) $30 \mu \mathrm{L}$ TFA (c) $30 \mu \mathrm{L}$ TFA $+40 \mu \mathrm{L}$ triethylamine

\section{3 结论}

本文合成了一种含腈基的芳基并吡咯化合物 DPPDC, 研究结果表明, 所合成的目标分子 DPPDC 具 有 AIE 特性, 聚集态的最大荧光强度相较于纯 THF 溶液 的苂光强度增强了 11 倍. 在不同的溶剂体系中共培养 得到四种不同的单晶结构, 晶体中分子构象也完全不 同，它们最大苂光发射波长分别为 467, 483, 493 和 496 $\mathrm{nm}$. 同时, 由于并吡咯核富含电子, DPPDC 能与三氟乙 酸相互作用, 使 DPPDC 的苂光发射发生明显变化, 由 黄色转为橙红色, 在三乙胺熏蒸的作用下, DPPDC 的苂 光发射又能恢复到黄色, 故该物质有望用于酸气体的可 视化检测.

\section{4 实验部分}

\subsection{1,4-双(4-溴苯基)-2,5-二苯基-1,4-二氢吡咯并 [3,2-b]吡咯(1)}

将 $1.02 \mathrm{~mL}(10 \mathrm{mmol})$ 的苯甲酫、 $1.72 \mathrm{~g}(10 \mathrm{mmol})$ 的对溴苯胺、 $0.172 \mathrm{~g}$ (1 mmol)的对甲基苯磺酸加入到 10 $\mathrm{mL}$ 的乙酸中, 摚拌并加热到 $90{ }^{\circ} \mathrm{C}$ 保持 $30 \mathrm{~min}$, 再向其 中缓慢滴加 $0.44 \mathrm{~mL}(5 \mathrm{mmol})$ 的丁二酮, 恒温保持 $3 \mathrm{~h}$. 反应结束后冷却到室温, 过滤得到黄色沉淀, 经柱色谱
提纯(用二氯甲烷/石油醚 $=1 / 4, V / V)$, 旋干得到浅黄色 固体粉末 $0.31 \mathrm{~g}$, 产率 $11 \% .{ }^{1} \mathrm{H} \mathrm{NMR}\left(400 \mathrm{MHz}, \mathrm{CDCl}_{3}\right)$ $\delta: 7.52 \sim 7.54(\mathrm{~m}, 4 \mathrm{H}), 7.19 \sim 7.30(\mathrm{~m}, 14 \mathrm{H}), 6.44(\mathrm{~s}, 2 \mathrm{H})$ (图 S5). MALDI-TOF $\mathrm{m} / \mathrm{z}$ : 理论值: 568.0 , 实测值: 568.3 (图 S6).

\subsection{4',4"-(2,5-二苯基吡咯并[3,2- $b]$ 吡咯-1,4-二基)双 ([1,1'-联苯]-4-腈)(DPPDC)}

将 $113.6 \mathrm{mg}(0.2 \mathrm{mmol})$ 的 $188.2 \mathrm{mg}(0.6 \mathrm{mmol})$ 的 4腈基苯硼酸、12 mg (0.01 mmol)的四三苯基磷钯, $135 \mathrm{mg}$ (1 mmol)的碳酸钾加入到 $100 \mathrm{~mL}$ 的两口圆底烧瓶, 抽真 空充氮气三次, 加入 $12 \mathrm{~mL}$ 除氧的溶剂(甲苯/甲醇 $=$ 3/1). 回流 $8 \mathrm{~h}$ 后, 冷却到室温, 水/二氯甲烷洗反应溶 液, 分离有机相, 无水硫酸镁干燥, 旋干, 用柱色谱提 纯 (二氯甲烷/乙酸乙酯/石油醚 $=1 / 1 / 6, V / V)$, 收集产物 点, 旋干得到浅黄色固体粉末 $30.3 \mathrm{mg}$, 产率 $24 \% .{ }^{1} \mathrm{H}$ NMR $\left(400 \mathrm{MHz}, \mathrm{CDCl}_{3}\right) \delta: 7.69 \sim 7.75(\mathrm{~m}, 8 \mathrm{H}), 7.59 \sim$ $7.61(\mathrm{~m}, 4 \mathrm{H}), 7.39 \sim 7.41(\mathrm{~m}, 4 \mathrm{H}), 7.20 \sim 7.28(\mathrm{~m}, 10 \mathrm{H})$, $6.50(\mathrm{~s}, 2 \mathrm{H})$ (图 S7). MALDI-TOF $m / z$ : 理论值: 612.2 , 实 测值: 612.5 (图 S8).

\section{References}

[1] (a) Kim, S.; Kim, B.; Lee, J.; Shin, H.; Park, Y.; Park, J. Mat. Sci. Eng. R 2016, 99, 1; (b) ) Zhao, Z.; Li, Z.; Lam, J. W. Y.; Maldonado, J. L.; Ramos-Ortiz, G.; Liu, Y.; Yuan, W.; Xu, J.; Miao, Q.; Tang, B. Z. Chem. Commun. 2011, 47, 6924; (c) Fujisawa, K.; Okuda, Y.; Izumi, Y.; Nagamatsu, A.; Rokusha, Y.; Sadaike, Y.; Tsutsumi, O. J. Mater. Chem. C 2014, 2, 3549; (d) Zhao, N.; Zhang, C.; Lam, J. W. Y.; Zhao, Y. S.; Tang, B. Z. Asian J. Org. Chem. 2014, 3, 118; (e) Mukherjee, S.; Thilagar, P. J. Mater. Chem. C 2016, 4, 2647; (f) Pan, L. X.; Luo, W. W.; Chen, M.; Liu, J. K.; Xu, L.; Hu, R. R.; Zhao, Z. J.; Qin, A. J.; Tang, B. Z. Chin. J. Org. Chem. 2016, 36, 1316. (潘凌 翔, 罗文文, 陈明, 刘峻恺, 徐露, 胡蓉蓉, 赵祖金, 秦安军, 唐 本忠, 有机化学, 2016, 36, 1316.)

[2] Wang, K.; Zhang, H. Y.; Chen, S. Y.; Yang, G. C.; Zhang, J. B.; Tian, W. J.; Su, Z. M.; Wang, Y. Adv. Mater. 2014, 26, 6168.

[3] Dong, Y. J.; Xu, B.; Zhang, J. B.; Tan, X.; Wang, L. J.; Chen, J. L.; Lv, H. G.; Wen, S. P.; Li, B.; Ye, L.; Zou, B.; Tian, W. J. Angew. Chem. Int. Ed. 2012, 51, 10782.

[4] Yoon, S. J.; Chung, J. W.; Gierschner, J.; Kim, K. S.; Choi, M. G.; Kim, D.; Park, S. Y. J. Am. Chem. Soc. 2010, 132, 13675.

[5] Luo, J.; Xie, Z.; Lam, J. W. Y.; Cheng, L.; Chen, H.; Qiu, C.; Kwok, H. S.; Zhan, X.; Liu, Y.; Zhu, D.; Tang, B. Z. Chem. Commun. 2001, 1740 .

[6] (a) Li, K.; Qin, W.; Ding, D.; Tomczak, N.; Geng, J.; Liu, R.; Liu, J.; Zhang, X.; Liu, H.; Liu, B. Sci. Rep. 2013, 3, 1150; (b) Wang, D.; Qian, J.; Qin, W.; Qin, A.; Tang, B. Z.; He, S. Sci. Rep. 2014, 4, 4279; (c) Sun, J. B.; Zhang, G. H.; Jia, X. Y.; Xue, P. C.; Jia, J. H.; Lu, R. Acta Chim. Sinica 2016, 74, 165. (孙静波，张恭贺，贾小宇， 薛鹏冲, 贾俊辉, 卢然, 化学学报, 2016, 74, 165.); (d) Wang, C.; Zhang, H.; Tian, L.; Zhu, W.; Lan, Y.; Li, J.; Wang, H.; Zhang, G. X. Zhang, D. Q.; Yuan, S. L.; Li, G. T. Sci. China Chem. 2016, 59, 89; (e) Zhao, Z.; Lam, J. W. Y.; Tang, B. Z. Curr. Org. Chem. 2010, 14, 2109; (f) Gu, X. G.; Yao, J. J.; Zhang, G. X.; Zhang, C.; Yan, Y. L.; Zhao, Y. S.; Zhang, D. Q. Chem. - Asian J. 2013, 8, 2362; (g) Li, Z. Z.; Huo, Y. P.; Yang, X. H.; Ji, S. M. Chin. J. Org. Chem. 2016, 36, 2317. (李宗植, 霍延平, 阳香华, 籍少敏, 有机化学, 2016, 36, 2317.)

[7] Mei, J.; Hong, Y. N.; Lam, J. W. Y.; Qin, A. J.; Tang, Y. H.; Tang, B. Z. Adv. Mater. 2014, 26, 5429.

[8] He, Z. K.; Zhang, L. Q.; Mei, J.; Zhang, T.; Lam, J. W.Y.; Shuai, Z. G.; Dong, Y. Q.; Tang, B. Z. Chem. Mater. 2015, 27, 6601. 
[9] Xu, Y. X.; Wang, K.; Zhang, Y. J.; Xie, Z. Q.; Zou, B.; Ma, Y. G. J. Mater. Chem. C 2016, 4, 1257.

[10] Zhang, Z. Y.; Song, X. X.; Wang, S. P.; Li, F.; Zhang, H. Y.; Ye, K. Q.; Wang, Y. J. Phys. Chem. Lett. 2016, 7, 1697.

[11] Krzeszewski, M.; Thorsted, B.; Brewer, J.; Gryko, D. T. J. Org. Chem. 2014, 79, 3119.

[12] Hisaki, I.; Sakamoto, Y.; Shigemitsu, H.; Tohnai, N.; Miyata, M. Cryst. Growth Des. 2009, 9, 414.
[13] Janiga, A.; Glodkowska-Mrowka, E.; Stoklosa, T.; Gryko, D. T. Asian J. Org. Chem. 2013, 2, 411.

[14] Peng, Z.; Feng, X.; Tong, B.; Chen, D. D.; Shi, J. B.; Zhi, J. G.; Dong, Y. P. Sensor Actuat. B 2016, 232, 264.

[15] Zhu, X. L.; Huang, H.; Liu, R.; Jin, X. D.; Li, Y. H.; Wang, D. F.; Wang, Q.; Zhu, H. J. J. Mater. Chem. C 2015, 3, 3774.

(Cheng, B.) 